\title{
Demand Response Using Service Curves
}

\author{
Jean-Yves Le Boudec, Fellow, IEEE, Dan-Cristian Tomozei, Member, IEEE
}

\begin{abstract}
Existing methods for demand response either assume direct control of appliances by supplier, or assume that consumers adapt their load by reacting to pricing signals. The former are intrusive and might not scale well; the latter expose consumers to price volatility and require detailed awareness of time varying prices. We propose an alternative approach, based on "service curves", which uses the following two ingredients. (1) The rate at which a consumer may draw power from the grid may be controlled by real time signals. Typical concerned devices would be heating systems, air conditioners and e-car batteries. (2) However, consumers are guaranteed that, over any window of time $t$, the amount of energy that may be drawn is at least equal to $b(t)$, where the function $b()$ (called the service curve) is agreed upon by contract at subscription time. The contract also specifies the maximum power that may be drawn, as well as a fixed price per unit of energy. Hence users are protected from price variability, at the expense of possible, but upper bounded, delays. With a proper service curve definition, an operator may distribute small service reductions in order to alleviate the impact of massive incoming demand onto the power grid. Consumers are able to observe the past service control signals that they received and can compute optimal load schedules from this and their service curve contracts, using only local information. Thus, this provides a distributed, scalable and robust demand response mechanism.
\end{abstract}

Index Terms-Demand Response, Service Curves, Load Switches

\section{INTRODUCTION}

Demand response is often assumed to be triggered by pricing signals [1]. However, dynamic pricing comes with a number of issues, in particular, consumer exposure to large price volatility might be socially undesirable. Further, it has been shown [4] that, even in a honest market, dynamic pricing may result in high volatility on the energy market. An alternative to end consumer pricing is implemented in load switches or centralized remote control. For example, the Voltalis bluepod switches off thermal electrical loads (boiler, thermal electrical heating) [5, chapter 4]. When and for how long the load may be switched off is under the control of the Voltalis company, however, consumers receive the guarantee that such service inhibitions occur for a total duration of at most $30 \mathrm{mn}$ per day. Pricing is not involved, and consumers pay per unit of consumed energy. In short, consumers are exposed to delayed supply rather than price volatility.

In this paper we propose an extension of such a scheme, called "service curves"; it is more general, and could serve

Jean-Yves Le Boudec and Dan-Cristian Tomozei (email: firstname dot lastname at epfl dot ch) are with the École Polytechnique Fédérale de Lausanne, School of Computer and Communication Sciences, Laboratory for computer Communications and Applications, CH-1015 Lausanne, Switzerland.

Presented in the second European conference on Innovative Smart Grid Technologies (ISGT-EUROPE 2011), sponsored by the IEEE Power \& Energy Society (PES) and hosted by the School of Electrical and Electronic Engineering of The University of Manchester, December 5 - 7, 2011 at Manchester Central Complex in Manchester, United Kingdom. non thermal loads, in particular plug-in electric vehicle (PEV) loads. It is inspired by the service curve approach used in the integrated services Internet framework [3]. With our scheme, the control exercised on demand need not be On/Off, as it is with Voltalis's devices. We show in Section VI that this may have benefits for reducing variance of the load.

Unlike centralized remote control, the service curves scheme does not require distribution systems operators (DSOs) or market aggregators to directly interfere with consumers' appliances, it provides one level of separation between consumers and the distribution system.

The contributions of our paper are as follows.

1) We introduce the concept of service curves. This framework supports demand response without exposing consumers to price volatility, at the expense of some limited delay or throttle.

2) We show how the framework allows a $\mathrm{DSO}^{1}$ to exercise some control on demand in order to smoothen the load curve, or even delay some demands in case of stochastic load surge or supply reduction. In the case of random but stationary load, it is best for the DSO to send smooth control signals. In contrast, for reducing peak demand, it is best for the DSO to send binary signals. We also show how consumer appliances can compute online schedules using only locally observed information.

3) We relate the framework to max-plus calculus and derive rules and methods to define and implement service curve constraints.

We define our framework in Section II, and give two possible examples in Section III. In Section IV we discuss some properties of service curves. The implications for consumers and distribution systems operators are described in Sections V to VII.

\section{Definition of the Service Curve Approach}

We assume that the rate at which a consumer may draw power from the grid may be controlled through load control signals. Such signals can be captured by a smart home controller who then uses them to compute a schedule for this home's appliances. The scheduled appliances are those that may have some elasticity, for example heating systems with or without heat pumps, air conditioners and PEV batteries. Non elastic appliances would not be controlled. The controls can use cellular networks or the smart grid; protocols and communication systems required to implement them are outside the scope of this paper (see [2] for a description of such a system).

\footnotetext{
${ }^{1}$ Demand response may be exercised by the DSO or other players, such as market aggregators. In the context of this paper it is irrelevant to know which entity issues the control signal; we use the term DSO as a general concept for the entity that issues the load control signal $u(t)$ defined in Section II.
} 


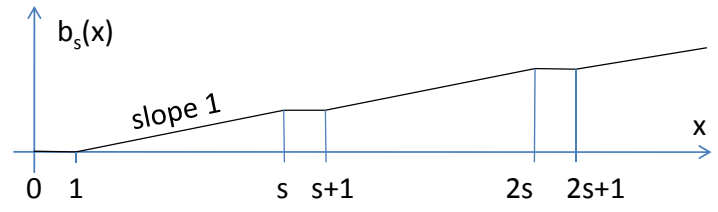

Fig. 1. The function $b_{s}$

Let $u(t)$ be the control signal received by one specific consumer and $z(t)$ the net power drawn by this consumer from the grid at time $t$ (in Watts). ${ }^{2}$ The contract between consumer and distribution system operator specifies the following constraints. First

$$
z(t) \leq u(t) \leq z_{\max } \text { for all } t^{\prime}<t
$$

i.e. the consumed energy may not exceed the level $u(t)$ specified by the distributor; $z_{\max }$ is the maximum that may ever be allowed.

Second, the consumer receives the guarantee that throttling cannot be excessive:

$$
\int_{t^{\prime}}^{t} u(\tau) d \tau \geq \beta\left(t-t^{\prime}\right) \text { for all } t^{\prime}<t
$$

where $\beta: \mathbb{N} \rightarrow[0,+\infty)$ is a function defined at contract subscription time. We say that $\beta$ is a service curve, and Eq. (2) can be paraphrased by saying that the control signals are constrained by the service curve $\beta$.

\section{EXAMPLES}

In this section we motivate and describe two examples of service definitions. Later in the paper, we compare the impact of these definitions on consumer perceived quality of service and on the amount of load smoothing that can be achieved by a distribution operator.

\section{(a) Load Switching}

It is convenient to introduce the function $b_{s}:[0,+\infty) \rightarrow$ $[0,+\infty)$ defined by

$$
\left\{\begin{array}{l}
b_{s} \text { is continuous and } b_{s}(0)=0 \\
\frac{\partial b_{s}(x)}{\partial x}=0 \text { for } 0<x<1, \quad \frac{\partial b_{s}(x)}{\partial x}=1 \text { for } 1<x<s \\
\frac{\partial b_{s}(x+s)}{\partial x}=\frac{\partial b_{s}(x)}{\partial x}
\end{array}\right.
$$

so that

$$
b_{s}(x) \stackrel{\text { def }}{=} \max (x,\lfloor x / s\rfloor s+1)-(\lfloor x / s\rfloor+1)
$$

where $\lfloor x\rfloor$ is the floor function, i.e. the largest integer $\leq x$ (see Figure 1). Voltalis's bluepod can be expressed in our framework with the service curve

$$
\beta_{1}(t)=t_{0} z_{\max } b_{t_{1} / t_{0}}\left(t / t_{0}\right)
$$

with $t_{0}=30 \mathrm{mn}$ and $t_{1}=24$ hours (see Figure 2).

One may think of this service curve constraint as the possibility to impose switch off periods for a total duration of

\footnotetext{
${ }^{2}$ We assume $z(t)$ is positive, i.e. we focus on controlling demand only. Extension of this framework to consumers who are also producers is for further study.
}

$30 \mathrm{mn}$ every 24 hours, while otherwise energy may be drawn at a rate equal to the power $z_{\max }$. However, the service curve constraint allows for more general controls than on-off; for example, we might impose a control $u(t)=z_{\max } / 2$ for $60 \mathrm{mn}$ instead of $u(t)=0$ for $30 \mathrm{mn}$; there are many combinations compatible with Eq. (2).

\section{(b) Two-Level Load Control}

The service curve $\beta_{1}$ in Eq. (4) and Figure 2 may be appropriate when the distribution system operator directly controls one or several appliances, but may not be so if we wish to leave such a control to the customer's smart home controller. For such cases, the control applies to the aggregate power drawn by one customer and it may be desirable to allow for a constant minimum power at any time (in order to serve non elastic appliances). Assume therefore that we want to define a service curve that (1) allows for power $z_{\max }$ except perhaps for $t_{0}$ hours every $t_{1}$ hours, but (2) also allows for a power $z_{\min }$ at any time. This corresponds to the following service curve:

$$
\beta_{2}(t)=z_{\min } t+\left(z_{\max }-z_{\min }\right) t_{0} b_{t_{1 / t_{0}}}\left(t / t_{0}\right)
$$

(See Figure 3). Note that the service curve $\beta_{1}$ is a special case of $\beta_{2}$ with $z_{\min }=0$.

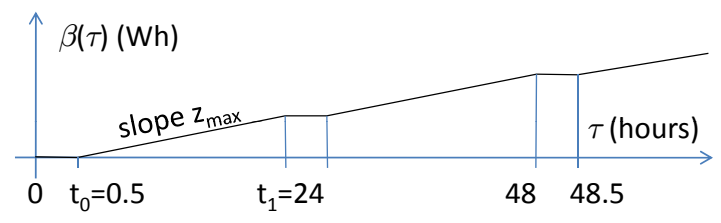

Fig. 2. The service curve $\beta_{1}$, shown here, allows the distributor to switch off the load for at most $30 \mathrm{mn}$ every day, or (for example) to reduce the load to $z_{\max } / 2$ for $60 \mathrm{mn}$ every day.

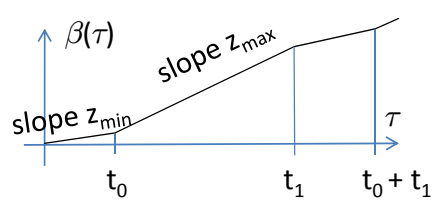

Fig. 3. The service curve $\beta_{2}$, shown here, corresponds to two-level load control, with minimum power $z_{\min }$ allowed at any time and maximum power $z_{\max }$ allowed at any time except perhaps for $t_{0}$ hours per period of $t_{1}$ hours.

\section{The Calculus of Service Curves}

For using service contracts as in Section III, it is important to understand how to design and implement service curve constraints. Since service curves define a maximum value over any sliding window, they should be super-additive $[3$, Section 3.1.8], which means that $\beta\left(t+t^{\prime}\right) \geq \beta(t)+\beta\left(t^{\prime}\right)$ for any nonnegative $t$ and $t^{\prime}$.

More precisely, if we define a contract using a service curve $\beta$ that is not super-additive, we obtain an equivalent definition if we replace $\beta$ by its super-additive closure $\bar{\beta}$, which is 
defined as the smallest super-additive function lower-bounded by $\beta$. Since $\bar{\beta} \geq \beta$ we have an apparently more stringent constraint [3, Theorem 3.1.10]. Note that the functions $\beta_{1}$ and $\beta_{2}$ introduced in Section III are super-additive, as is the function $b_{s}$ (as we show in the proof of Lemma 1).

This has the following implication. If $\beta(t)=z_{0} t$ for $0 \leq$ $t \leq t_{0}$ for some positive $t_{0}$ and $z_{0}$ (i.e. we want to allow for some power $z_{0}$ over some period of time), then the superadditive closure satisfies $\bar{\beta}(t) \geq z_{0} t$ for all $t \geq 0$ (i.e. we must guarantee $z_{0}$ over any period of time).

Also, assume that we want to design a service contract that several several constraints, such as

$$
\begin{aligned}
& \int_{t^{\prime}}^{t} u(\tau) d \tau \geq \beta\left(t-t^{\prime}\right) \\
& \int_{t^{\prime}}^{t} u(\tau) d \tau \geq \beta^{\prime}\left(t-t^{\prime}\right)
\end{aligned}
$$

for all $t^{\prime}<t$. Then we must in fact impose a service curve equal to the super-additive closure of $\max \left(\beta, \beta^{\prime}\right)$, which, in general, is not the same as $\max \left(\beta, \beta^{\prime}\right)$. For example, we will see in Lemma 1 that the service curve $\beta_{2}$ in Figure 3 is equivalent to simultaneously imposing the two constraints $\beta_{2}^{\prime}(t)=z_{\min } t$ and $\beta_{2}^{\prime \prime}(t)=t_{0}^{\prime} z_{\max } b_{t_{1} t_{0}^{\prime}}\left(t / t_{0}^{\prime}\right)$ with $t_{0}^{\prime}=$ $\left(1-z_{\min } / z_{\max }\right) t_{0}$.

In the rest of this section we use these results to propose practical ways to implement the service curves $\beta_{1}$ and $\beta_{2}$ introduced in Section III.

\section{(a) Load Switching}

The following theorem shows that the service curve constraint $\beta_{1}$ can be implemented by counting the control signals over sliding windows of duration $t_{1}$ :

Theorem 1. Let $u(t)$ be a sequence of control signals defined up to some time horizon $T$. Assume that $u(t) \leq z_{\max }$ for all $t \leq T$. The two properties are equivalent:

(i) $\int_{t^{\prime}}^{t} u(\tau) d \tau \geq \beta_{1}\left(t-t^{\prime}\right)$ for all $t^{\prime}<t \leq T$, where $\beta_{1}$ is the service curve in Eq. (4).

(ii) $\int_{t}^{t+t_{1}} u(\tau) d \tau \geq z_{\max }\left(t_{1}-t_{0}\right)$ for all $t$ such that $t+t_{1} \leq$ $T$.

Proof: (i) $\Rightarrow$ (ii) is immediate (take $t=t^{\prime}+t_{1}$ and note that $\left.\beta_{1}\left(t_{1}\right)=z_{\max }\left(t_{1}-t_{0}\right)\right)$.

(ii) $\Rightarrow$ (i) First we show an alternative representation for $\beta_{1}$. Let $t \geq 0$ and let $q$ be the quotient in the euclidian division of $t$ by $t_{1}$, and $r$ the remainder. In other words, $q=\left\lfloor\frac{t}{t_{1}}\right\rfloor$ and $r=t-q t_{1}$. We claim that

$$
\beta_{1}(t)=q \beta_{1}\left(t_{1}\right)+z_{\max }\left(r-t_{0}\right)^{+}
$$

where the notation $x^{+}$means $\max (x, 0)$. This can easily be shown by induction on $q$.

Second, for any nonnegative integer $k$, we claim that

$$
\int_{t^{\prime}}^{t^{\prime}+k t_{1}} u(\tau) d \tau \geq k \beta_{1}\left(t_{1}\right)
$$

To see why, note that

$$
\begin{aligned}
\int_{t^{\prime}}^{t^{\prime}+k t_{1}} u(\tau) d \tau= & \int_{t^{\prime}}^{t^{\prime}+t_{1}} u(\tau) d \tau+\ldots \\
& +\int_{t^{\prime}+(k-1) t_{1}}^{t^{\prime}+k t_{1}} u(\tau) d \tau \\
\geq & k \beta_{1}\left(t_{1}\right)=\beta_{1}\left(k t_{1}\right)
\end{aligned}
$$

where the last equality is by Eq. (6).

Third, take some arbitrary $t^{\prime}<t$ and let $q=\left\lfloor\frac{t-t^{\prime}}{t_{1}}\right\rfloor, r=$ $t-t^{\prime}-q t_{1}$. Assume first that $t_{0} \leq r$. Then:

$$
\begin{aligned}
\int_{t^{\prime}}^{t} u(\tau) d \tau & =\int_{t^{\prime}}^{t^{\prime}+(q+1) t_{1}} u(\tau) d \tau-\int_{t}^{t^{\prime}+(q+1) t_{1}} u(\tau) d \tau \\
& \geq(q+1) \beta_{1}\left(t_{1}\right)-z_{\max }\left(t_{1}-r\right)
\end{aligned}
$$

where the former term is by Eq. (7) and the latter because $u(\tau) d \tau \leq z_{\max }$ by hypothesis. Recall that $\beta_{1}\left(t_{1}\right)=z_{\max }\left(t_{1}-\right.$ $\left.t_{0}\right)$, it follows that

$$
\begin{aligned}
\int_{t^{\prime}}^{t} u(\tau) d \tau & \geq q \beta_{1}\left(t_{1}\right)+z_{\max }\left(t_{1}-t_{0}\right)-z_{\max }\left(t_{1}-r\right) \\
& =q \beta_{1}\left(t_{1}\right)+z_{\max }\left(r-t_{0}\right)=\beta_{1}\left(t-t^{\prime}\right)
\end{aligned}
$$

where we used Eq. (6) and the condition $t_{0} \leq r$.

Consider now the case where $t_{0}>r$. In this case $\beta_{1}(t-$ $\left.t^{\prime}\right)=q \beta_{1}\left(t_{1}\right)$. Further, $t \geq t^{\prime}+q t_{1}$ thus

$$
\begin{aligned}
\int_{t^{\prime}}^{t} u(\tau) d \tau & \geq \int_{t^{\prime}}^{t^{\prime}+q t_{1}} u(\tau) d \tau \\
& \geq q \beta_{1}\left(t_{1}\right)=\beta_{1}\left(t-t^{\prime}\right)
\end{aligned}
$$

Theorem 1 gives a practical method for enforcing or verifying the service curve constraint $\beta_{1}$. It is sufficient to keep in memory the history of the control signals over the last $t_{1}$ time units; their integral should never drop below $z_{\max }\left(t_{1}-t_{0}\right)$.

\section{(b) Two-Level Load Control}

We have a similar theorem for the service curve $\beta_{2}$. Recall that $\beta_{1}$ is a special case of $\beta_{2}$ with $z_{\text {min }}=0$, therefore Theorem 1 can be viewed as a consequence of Theorem 2 . However the proof of Theorem 2 uses Theorem 1, which explains why we present the theorems in this order. We start with a lemma:

Lemma 1. A sequence of control signals is constrained by the service curve $\beta_{2}$ in Eq. (5) if and only if it is simultaneously constrained by each of the two service curves defined by

$$
\begin{aligned}
& \beta_{2}^{\prime}(t)=z_{\min } t \\
& \beta_{2}^{\prime \prime}(t)=t_{0}^{\prime} z_{\max } b_{t_{1} / t_{0}^{\prime}}\left(t / t_{0}^{\prime}\right) \text { with } t_{0}^{\prime}=\left(1-z_{\min } / z_{\max }\right) t_{0}
\end{aligned}
$$

Proof: (i) We show that $b_{s}$ is super-additive. Note that $b_{s}(t)=q(s-1)+(r-1)^{+}$as in Eq. (6) where $q$ is the quotient in the euclidean division of $t$ by $s$, and $r$ the remainder. Thus for any nonnegative integer $k$ :

$$
b_{s}(t+k s)=k(s-1)+b_{s}(t)
$$


Thus for any any nonnegative integers $k, k^{\prime}$ :

$$
\begin{aligned}
& b_{s}\left(t+t^{\prime}+\left(k+k^{\prime}\right) s\right)-b_{s}(t+k s)-b_{s}\left(t+k^{\prime} s\right)= \\
& b_{s}\left(t+t^{\prime}\right)-b_{s}(t)-b_{s}\left(t^{\prime}\right)
\end{aligned}
$$

and it is sufficient to show

$$
b_{s}\left(t+t^{\prime}\right) \geq b_{s}(t)+b_{s}\left(t^{\prime}\right) \text { for } 0 \leq t<s \text { and } 0 \leq t^{\prime}<s
$$

This is easily done by considering the four cases obtained by testing $t<1$ and $t^{\prime}<1$ (with $0 \leq t<s$ and $0 \leq t^{\prime}<s$ ).

(ii) We show that $\beta_{2}$ is super-additive. This follows directly from the representation

$$
\beta_{2}(t)=z_{\min } t+t_{0}\left(z_{\max }-z_{\min }\right) b_{t_{1} / t_{0}}\left(t / t_{0}\right)
$$

and from (i).

(iii) We show that $\beta_{2}$ is equal to the super-additive closure $\bar{\beta}^{\prime}$ of $\beta^{\prime}=\max \left(\beta_{2}^{\prime}, \beta_{2}^{\prime \prime}\right)$. Note that $\beta_{2} \geq \beta_{2}^{\prime}$ and $\beta_{2} \geq \beta_{2}^{\prime \prime}$ thus $\beta_{2} \geq \beta^{\prime}$ and since $\beta_{2}$ is super-additive, $\beta_{2} \geq \bar{\beta}^{\prime}$. Now we show that $\beta_{2} \leq \bar{\beta}^{\prime}$.

Note that $\beta_{2}^{\prime}$ and $\beta_{2}^{\prime \prime}$ are super-additive (this follows from (i)), thus [3, Theorem 3.1.11] the super-additive closure of $\beta^{\prime}$ is $\beta_{2}^{\prime} \bar{\otimes} \beta_{2}^{\prime \prime}$, where $\bar{\otimes}$ denotes the max-plus convolution:

$$
\left(\beta_{2}^{\prime} \bar{\otimes} \beta_{2}^{\prime \prime}\right)(t)=\sup _{t^{\prime} \leq t}\left(\beta_{2}^{\prime}\left(t^{\prime}\right)+\beta_{2}^{\prime \prime}\left(t-t^{\prime}\right)\right)
$$

Therefore we need to show that for all nonnegative $t$ there exists some nonnegative $t^{\prime} \leq t$ such that

$$
\beta_{2}(t) \leq \beta_{2}^{\prime}\left(t^{\prime}\right)+\beta_{2}^{\prime \prime}\left(t-t^{\prime}\right)=z_{\min } t^{\prime}+\beta_{2}^{\prime \prime}\left(t-t^{\prime}\right)
$$

Let $q$ be the quotient in the euclidean division of $t$ by $t_{1}$, and $r$ the remainder. If $r \geq t_{0}$ then $\beta_{2}(t)=\beta_{2}^{\prime \prime}(t)$ and Eq. (9) is satisfied with $t^{\prime}=0$. Else, $\beta_{2}(t)=\beta_{2}^{\prime \prime}\left(q t_{1}\right)+z_{\min } r$ and Eq. (9) is satisfied with $t^{\prime}=r$.

(iv) We are now in a position to prove the statement in the lemma. Note that $\beta_{2} \geq \beta_{2}^{\prime}$ and $\beta_{2} \geq \beta_{2}^{\prime \prime}$ therefore the "only if" part of the theorem is obvious. Assume now that a sequence of control signals $u(\tau)$ is constrained by the service curves $\beta_{2}^{\prime}$ and $\beta_{2}^{\prime \prime}$. It is therefore constrained by $\beta^{\prime}=\max \left(\beta_{2}^{\prime}, \beta_{2}^{\prime \prime}\right)$ and, therefore by the super-additive closure of $\beta^{\prime}$, which by (iii) is equal to $\beta_{2}$.

Theorem 2. Let $u(t)$ be a sequence of control signals defined up to some time horizon $T$. Assume that $u(t) \leq z_{\max }$ for all $t \leq T$. The two properties are equivalent:

(i) $\int_{t^{\prime}}^{t} u(\tau) d \tau \geq \beta_{2}\left(t-t^{\prime}\right)$ for all $t^{\prime}<t \leq T$, where $\beta_{2}$ is the service curve in Eq. (5).

(ii) $u(\tau) \geq z_{\min }$ for all $\tau \leq T$,

and $\int_{t}^{t+t_{1}} u(\tau) d \tau \geq z_{\max } t_{1}-\left(z_{\max }-z_{\min }\right) t_{0}$ for all $t$ such that $t+t_{1} \leq T$.

Proof: (i) $\Rightarrow$ (ii) is immediate as in the proof of Theorem 1, after noticing that $\beta_{2}\left(t_{1}\right)=z_{\max } t_{1}-\left(z_{\max }-z_{\min }\right) t_{0}$.

(ii) $\Rightarrow$ (i) By Lemma 1, it is sufficient to show that the constraints with service curves $\beta_{2}^{\prime}$ and $\beta_{2}^{\prime \prime}$ are both satisfied. The constraint with service curve $\beta_{2}^{\prime}$ is obviously satisfied because $u(\tau) \geq z_{\min }$ for every $\tau$. For the second constraint, note that $\beta_{2}^{\prime \prime}$ is the same as $\beta_{1}$ if we replace $t_{0}$ by $t_{0}^{\prime}=\left(1-z_{\min } / z_{\max }\right) t_{0}$ and that $z_{\max } t_{1}-\left(z_{\max }-z_{\min }\right) t_{0}=z_{\max }\left(t_{1}-t_{0}^{\prime}\right)$. Then apply Theorem 1 .
Theorem 2 gives a practical method for enforcing or verifying the service curve constraint $\beta_{2}$. It is sufficient to check that the control signals satisfy $u(\tau) \geq z_{\min }$ at every time slot $\tau$ and to keep in memory the history of the control signals over the last $t_{1}$ time units; their integral should never drop below $z_{\max } t_{1}-\left(z_{\max }-z_{\min }\right) t_{0}$. Note that the constraint $u(\tau) \leq z_{\max }$ is always implicitly enforced, since we assume that the consumed power can never exceed $z_{\max }$.

\section{Consumer Side Problem}

In this section we illustrate how a consumer may optimize her use of a service contract with service curve. Assume a consumer wants to recharge her PEV battery, using a power plug controlled by a smart home controller, with a contract as defined above, with service curve $\beta_{2}$. Assume the minimum guaranteed power $z_{\min }$ can be used at any time by other, non elastic appliances. Assume the PEV is the only elastic appliance (the more general problem involves scheduling of multiple elastic appliances by the smart home controller and is left for further study). Assume the consumer is interested in having a fully charged battery at time horizon $T$, given that at time 0 the current charge level is $B(0)$. Since the contract specifies a fixed price per unit of energy, she is interested in minimizing the consumed power, subject to feasibility. In other words, she wants to have a battery fully loaded at time $T$; if this is possible, she further wants to minimize the electricity cost. Let $B(t)$ be the battery level at time $t$, and $B_{\max }$ the full battery level. We can express the consumer's problem as the desire to minimize the cost function

$$
K\left(B_{\max }-B(T)\right)+\int_{0}^{T} z(\tau) d \tau
$$

where $K$ is a large constant $\left(K>T z_{\max }\right)$. The equations for battery level are

$$
B(t)-B(0)=\int_{0}^{t}\left(\eta z(\tau)-\rho z(\tau)^{2}-\gamma \mathbb{1}_{\{B(\tau)>0\}}\right) d \tau
$$

where the notation $\mathbb{1}_{\{\text {condition }\}}$ means 1 when condition is true and 0 otherwise, and

$$
\begin{aligned}
& \eta=\text { efficiency } \\
& \gamma=\text { battery leakage } \\
& \rho=\text { thermal loss }
\end{aligned}
$$

Further, the load $z(t)$ must be such that $B(t) \leq B_{\max }$ at all times $t$.

The consumer's problem must be solved online; to make the problem tractable, we discretize time. The consumer's smart home controller needs to decide at time $t$ for a value $z(t+1)$, not knowing values of the future control signals $u(\tau), \tau>t$, using a "policy" $\pi$. The policy must be causal, i.e. decision taken by $\pi$ at time $t$ depends on $z(\tau), u(\tau)$ for $\tau \leq t$ and on the battery level $B(t)$. Further, the service curve contract must be obeyed, i.e. the policy must choose a load $z(t+1)$ such that

$$
z(t+1)+n(t+1) \leq u(t+1)
$$

where $n(t+1)$ is the non elastic load at time $t+1$. In this paper, we assume that the non-elastic load cannot be forecast 
or controlled, however it may not exceed $z_{\min }$. We assume therefore that the policy enforces the constraint

$$
z(t+1) \leq u(t+1)-z_{\min }
$$

which indeed allows non-elastic load to operate without hindrance.

The future control signals $u(t+1)$ are not known either at the time of decision making, but their worst case can be predicted, depending on the past. We also assume that the consumer's goal is to minimize the worst case total cost. In other words, let $C(\pi, u)$ be the total cost, given by Eq. (10) ( $u$ denotes the complete control signal for all times up to time $T)$. The goal is to find a policy $\pi$ that minimizes

$$
\max _{u} C(\pi, u)
$$

where the maximum is over all possible control signals that are compatible with the service curve constraint.

Finding an optimal policy seems to be an open problem and is left for further study. However we can provide some preliminary insight. The first question that can be addressed is whether a close to full charge can be achieved within the time horizon $T$. Note that a full charge cannot be guaranteed, since control signals sent towards the end of the charging period may stop the charging process, and some battery leakage is assumed in our model. Also note that the thermal loss term in Eq. (11) may imply that for large enough load, e.g., for $z(\tau)>\eta / \rho$, the net charge decreases; clearly, the battery charging system should not operate in this region, it is sufficient to assume that $z_{\max }-z_{\min }<\eta / 2 \rho$, with batteries such that $\eta^{2}>4 \gamma \rho$. We also assume that the horizon $T$ is less than $t_{1}$ (this is reasonable if we assume, as we did earlier, that $t_{1}$ is of the order of 24 hours).

Theorem 3. Consider the consumer side problem described earlier. Let $B(0)<B_{\max }$ be the initial battery level and $u(t), t=0,-1,-2, \ldots$ the past control signals (known to the consumer).

Let $V^{*}$ be the optimal value of the convex programming problem:

$$
\begin{aligned}
& \text { minimize } V=\sum_{\tau=1}^{T}\left(\eta x(\tau)-\rho x(\tau)^{2}\right) \\
& \text { over the variables } x(1), \ldots, x(T) \\
& \text { subject to the constraints } \\
& \left\{\begin{array}{c}
0 \leq x(\tau) \leq z_{\max }-z_{\min } \text { for } 1 \leq \tau \leq T, \\
\sum_{\tau=t+1}^{t+t_{1}} x(\tau) \geq\left(z_{\max }-z_{\min }\right)\left(t_{1}-t_{0}\right) \\
\text { for } 1-t_{1} \leq t \leq T-t_{1}
\end{array}\right.
\end{aligned}
$$

If $V^{*} \geq B_{\max }-B(0)+T \gamma$, there exists a policy $\pi$ that achieves $B(T) \geq B_{\max }-\gamma T$. This policy achieves full battery level at some time $t \in[0, T]$.

Proof: Let $u(t)$ be the actual value of the controls (not known in advance). Let $x(t)=u(t)-z_{\min }$ and let $B^{\prime}(t)$ be the level of a virtual battery, that would start at $B(0)$, would receive the load $x(t)$, would have 0 leakage, and infinite maximum level. In other words, $B^{\prime}(0)=B(0)$ and for $t=0, \ldots, T-1$ :

$$
B^{\prime}(t+1)-B^{\prime}(t)=\eta x(t)-\rho x(t)^{2}
$$

The sequence $x(t)$ satisfies the constraints in the theorem, therefore

$$
\begin{aligned}
B^{\prime}(T)-B^{\prime}(0) & =\sum_{\tau=1}^{T}\left(\eta x(\tau)-\rho x(\tau)^{2}\right) \\
& \geq V^{*} \geq B_{\max }-B(0)+T \gamma
\end{aligned}
$$

and thus

$$
B^{\prime}(T) \geq B_{\max }+T \gamma
$$

Now define the policy $\pi$ by

$$
\begin{aligned}
& z(t+1)=u(t+1)-z_{\min } \text { if } B(t) \leq B_{\max } \\
& z(t+1)=\min (u(t+1), \gamma) \text { if } B(t)=B_{\max }
\end{aligned}
$$

i.e. we set the consumed power $z(t)$ to the maximum possible considering the constraint of non elastic load and of maximum battery capacity. Let $B(t)$ be the battery level with this policy. As long as $B(t)<B_{\max }$, the rate at which $B$ and $B^{\prime}$ vary differ by at most the leakage term $\gamma$, thus as long as $B(t)<$ $B_{\max }$ :

$$
B^{\prime}(t)-\gamma t \leq B(t) \leq B^{\prime}(t)
$$

Assume that $B(t)$ never reaches $B_{\max }$. It follows that $B^{\prime}(T) \leq$ $B(T)+\gamma T<B_{\max }+\gamma T$, which contradicts Eq. (12), thus there exists some time $t$ at which $B(t)=B_{\max }$. The rest of the theorem follows from the fact that leakage occurs at rate $\gamma$.

\section{A Minimum Variance Policy (MCAP)}

In this and the next section we consider two scenarios where DSOs could make use of the service curve approach. In this section, we consider a case where load is random but stationary, and we study how the variance of the load can be reduced by using appropriate control signals. Reducing the variance reduces DSOs' hedging costs. The scenario considered here is highly idealized scenario, but it serves the purpose of showing that simple binary policies are not always optimal.

Consider an infinite population of identical customers having contracts specified by the service curve $\beta_{2}(\cdot)$. Assume that customers arrive according to a Poisson process of constant rate $\lambda$ with arrival times $\{S(n), n \in \mathbb{Z}\}$, consume an amount $\beta_{2}(T)$ of energy, for a fixed $T$ satisfying $t_{0}<T<t_{1}$, and subsequently leave the system forever. The quantity of energy consumed by each customer can be written as

$$
w:=\beta_{2}(T)=z_{\max } T-\left(z_{\max }-z_{\min }\right) t_{0} .
$$

The system operator chooses signal $u(s)$ satisfying the contract agreement

$$
\int_{t^{\prime}}^{t} u(s) d s \geq \beta_{2}\left(t-t^{\prime}\right), \forall t^{\prime}<t,
$$

that it sends to each customer upon arrival. Customers behave greedily and immediately consume their $w$ units of energy, as soon as the control signals allow them to do so. Denote the instantaneous power consumption of a customer at $t$ time units after her arrival in the system by

$$
z(t):=u(t) \mathbb{1}_{0 \leq t \leq T} .
$$




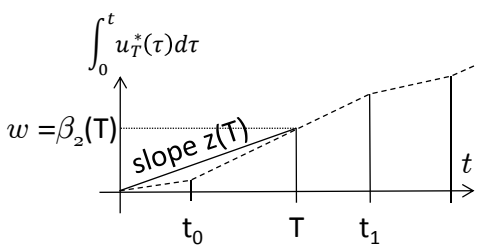

Fig. 4. Cumulative control $\int_{0}^{t} u_{T}^{*}(\tau) d \tau$ for the optimal policy $u_{T}^{*}$. The time $t=0$ is a customer arrival time.

Assume that the DSO knows $w$. If $\int_{0}^{T} z(s) d s<w$, then the contract is violated. On the other hand, if $\int_{0}^{T} z(s) d s>w$, then the DSO exceeds its obligations. Hence, the DSO wishes to have

$$
\int_{0}^{T} z(s) d s=\beta_{2}(T) .
$$

The aggregate instantaneous energy consumption observed by the DSO is given by the stochastic process

$$
Z(t)=\sum_{n=-\infty}^{+\infty} z(t-S(n)) .
$$

By Campbell's theorem, the mean and variance of $Z(t)$ are given by

$$
\begin{aligned}
\mathbb{E} Z & =\lambda \int_{-\infty}^{+\infty} z(s) d s=\lambda w, \\
\operatorname{Var}(Z) & =\lambda \int_{-\infty}^{+\infty} z^{2}(s) d s .
\end{aligned}
$$

Since the users have the same energy consumption, the DSO wishes to minimize the variance of the process $Z(t)$, i.e., to solve the following problem:

$$
\begin{aligned}
& \text { Minimize } \int_{-\infty}^{+\infty} z^{2}(s) d s \text { over } u(\cdot) \leq z_{\max } \\
& \text { such that } \int_{t^{\prime}}^{t} u(s) d s \geq \beta_{2}\left(t-t^{\prime}\right), \quad \forall 0 \leq t^{\prime}<t \leq T .
\end{aligned}
$$

A first choice is to take binary control $u(\cdot)$ equal to

$$
u_{b}(t)= \begin{cases}z_{\min }, & 0<t \leq t_{0} \\ z_{\max }, & \text { otherwise }\end{cases}
$$

a choice which, by Theorem 2, satisfies the constraint (16). By Jensen's inequality, we can write

$$
\begin{aligned}
\int_{0}^{T} u_{b}^{2}(s) d s & =t_{0} z_{\min }^{2}+\left(T-t_{0}\right) z_{\max }^{2} \\
& \geq T\left(\frac{t_{0}}{T} z_{\min }+\frac{T-t_{0}}{T} z_{\max }\right)^{2} .
\end{aligned}
$$

Denote

$$
z(T):=\frac{t_{0}}{T} z_{\min }+\frac{T-t_{0}}{T} z_{\max },
$$

and define the policy

$$
u_{T}^{*}(t)= \begin{cases}z(T), & 0<t \leq T \\ z_{\max }, & \text { otherwise }\end{cases}
$$

By Theorem 2, $u_{T}^{*}$ also satisfies constraint (16) and furthermore achieves a lower variance, by (17). We shall refer to this policy as the Minimum Constant Allocation Policy (MCAP). Let us prove that MCAP is optimal.

Theorem 4. For identical customers with contract given by $\beta_{2}(\cdot)$ arriving according to a Poisson process of rate $\lambda$ and consuming the same amount of energy $w=\beta_{2}(T)$ for a fixed value $t_{0}<T<t_{1}$, the MCAP control signal $u_{T}^{*}$ minimizes the variance of the aggregate demand $Z(t)$. In other words, it achieves the optimum of (15)-(16).

Proof: For any control $u$, by Cauchy-Schwartz,

$$
\int_{0}^{T} u^{2}(s) d s \int_{0}^{T} 1 d s \geq\left(\int_{0}^{T} u(s) d s\right)^{2} .
$$

Thus, using (14), we obtain a lower bound on the variance of $Z(t)$ :

$$
\int_{-\infty}^{+\infty} z^{2}(s) d s \geq \frac{1}{T} \beta_{2}^{2}(T)
$$

Since

$$
\int_{0}^{T} u_{T}^{* 2}(s) d s=\frac{1}{T} \beta_{2}^{2}(T),
$$

we can conclude.

Service curves give freedom of control choice for the DSO. As discussed in Section III, service curves such as $\beta_{2}(\cdot)$ allow the implementation of binary controls $u_{b}$ (which are similar to the ones implemented by Voltalis), but also of more complex ones. In the setting of steady arrival of identical users, we saw (Theorem 4) that binary controls are not the best suited for reducing peak consumption, and found that the smoother MCAP control $u_{T}^{*}$ achieves the goal, while satisfying the contract conditions.

In the next section we evaluate a heuristic for performing demand response using service curves in a setting of bursty arrivals.

\section{BURSTY ARRIVALS}

Like in Section VI, we consider consumers that have a guaranteed minimal service characterized by the service curve $\beta_{2}(\cdot)$. We have seen that, when arrivals are static and consumers homogeneous, the best policy for the system operator is to use MCAP, which imposes a constant power consumption for users. In this section, we define the Quota Binary Allocation Policy (QBAP), a heuristic which distributes temporary service reductions to the users of the system through binary power signals, while satisfying the contract specified via the service curve. We consider a setting in which arrivals are bursty. We show through numerical evaluations that QBAP outperforms MCAP in this scenario.

\section{A. Assumptions}

We consider a population of $N$ users $\mathcal{U}=\{1, \ldots, N\}$ with contracts specified by service curve $\beta_{2}(\cdot)$. At time $t$ each user $i \in \mathcal{U}$ receives control signal $u_{i}(t)$ from the DSO and consumes power $z_{i}(t)$, such that $z_{i}(t) \leq u_{i}(t)$.

We address a specific scenario where the $N$ consumers (or users) charge their PEV batteries upon arriving home after a work day. The arrival times are normally distributed and the 
car batteries are identical, ideal (i.e., no thermal losses, or leakage), have a maximum charging power $z_{b}$ and capacity $w$. Like in Section $\mathrm{V}$, consumers additionally impose a nonelastic load on the grid, which may not exceed $z_{\text {min }}$, and the PEV is their only elastic appliance. We set $t_{1}=24 \mathrm{~h}$ and $z_{\max }=z_{\min }+z_{b}$, such that it is possible to satisfy the nonelastic load and to charge the battery at maximum power for a duration of at least $t_{1}-t_{0}$ per day. The burst of consumption due to batteries causes the load on the grid to peak. We wish to design a policy which makes use of the freedom of decision provided by the service curve to reduce the peak load.

For presentation ease, in this section we consider discrete time. We note that the results presented in the previous sections directly apply for discrete time.

\section{B. Quota Binary Allocation Policy}

Theorem 2 states that in order to satisfy the constraints imposed by the service curve $\beta_{2}(\cdot)$, it is necessary and sufficient to guarantee an energy output of at least $z_{\max }\left(t_{1}-t_{0}\right)+z_{\min } t_{0}$ over any time window of length $t_{1}$, while at any time $t$, the control signal $u_{i}(t)$ sent to each user $i$ is such that $z_{\text {min }} \leq u_{i}(t) \leq z_{\max }$. We use this result to design a technique that enforces the service curve.

For each user $i$, fix $u_{i}(t)$ for the duration of time step $t$, and allow it to take only two possible values $\left\{z_{\min }^{\prime}, z_{\max }\right\}$, where $z_{\text {min }}^{\prime}=z_{\min }+\epsilon$, for a fixed $\epsilon>0$. We call $u_{i}(t)$ a discrete binary policy. We introduce $\epsilon$ for a signaling purpose: Since the non-elastic load is limited at $z_{\mathrm{min}}$, power consumption greater than $z_{\min }$ is an indication for the DSO that elastic load is present. Let us make sure that the control $u_{i}(t)$ satisfies the contract. By Theorem 2, it is sufficient to guarantee that $u_{i}(t)$ will be set to $z_{\text {min }}^{\prime}$ over no more than $t_{0}$ time steps within any time window of length $t_{1}$.

Setting $u_{i}(t)$ to $z_{\min }^{\prime}$ rather than $z_{\max }$ can be interpreted as a "penalty". Define a penalty budget sequence $\left(p_{i, t}\left(t^{\prime}\right)\right)_{t^{\prime} \geq 0}$ for each user $i$ and at each time step $t$. The values $p_{i, t}\left(t^{\prime}\right)$ represent the number of successive time steps, starting at time $t+t^{\prime}$, during which user $i$ can be limited at $z_{\min }$ without violation of the contract, if no low-power signals are sent between times $t$ and $t+t^{\prime}$. For example, if $p_{i, t}(1)=2$, then the control sequence $u_{i}(t . . t+3)=\left(z_{\max }, z_{\min }^{\prime}, z_{\min }^{\prime}, z_{\max }\right)$ is feasible, while the control sequence $u_{i}(t . . t+3)=\left(z_{\max }, z_{\min }^{\prime}, z_{\min }^{\prime}, z_{\min }^{\prime}\right)$ violates the penalty budget.

Implicitly, if $p_{i, t}(0) \geq 1$, then the control $u_{i}(t)$ may take any of the values $z_{\min }^{\prime}$ or $z_{\max }$, whereas if $p_{i, t}(0)=0$, then $u_{i}(t)$ must be set to $z_{\max }$. In the latter case, we say that the penalty budget of user $i$ is depleted.

Let us describe how the penalty budget sequence can be computed. By definition, $p_{i, t}\left(t^{\prime}\right)=t_{0}$, for all $t^{\prime} \geq t_{1}$. Therefore, the operator needs to maintain the penalty budget over a window of only $t_{1}$ time steps in the future: $\left(p_{i, t}(0), p_{i, t}(1), \ldots, p_{i, t}\left(t_{1}-1\right)\right)$.

At $t=0$ (i.e., no consumption history), for each user $i$, we have that $p_{i, t}\left(t^{\prime}\right)=t_{0}$, for all $t^{\prime} \geq 0$. At time $t$, the DSO can perform two operations: either send a maximum power signal $z_{\max }$ to user $i$, or, if the penalty budget allows it (i.e., $p_{i, t}(0) \geq 1$ ), a low-power signal $z_{\min }^{\prime}$. The operation performed by the DSO modifies the penalty budget sequence as follows:

- If $u_{i}(t)=z_{\max }$, then the penalty budget is not affected. Hence, at time step $t+1$ we have

$$
p_{i, t+1}\left(t^{\prime}\right)= \begin{cases}p_{i, t}\left(t^{\prime}+1\right), & 0 \leq t^{\prime}<t_{1}-1, \\ t_{0}, & t^{\prime} \geq t_{1}-1 .\end{cases}
$$

- If $p_{i, t}(0) \geq 1$, then the DSO may send a low-power signal to user $i, u_{i}(t)=z_{\min }^{\prime}$. In this case, the penalty budget is decremented and at time step $t+1$ we have:

$$
p_{i, t+1}\left(t^{\prime}\right)= \begin{cases}p_{i, t}\left(t^{\prime}+1\right)-1, & 0 \leq t^{\prime}<t_{1}-1 \\ t_{0}, & t^{\prime} \geq t_{1}-1\end{cases}
$$

In Algorithm 1 we propose a heuristic for reducing aggregate peak consumption, the Quota Binary Allocation Policy (QBAP). The DSO enforces the service curve using a sliding window of penalties $P_{i}$ of length $t_{1}$ for each user $i$, as described above. The algorithm takes as input a fixed "besteffort" quota $N_{a}>0$. At each time step, it aims for at most $N_{a}$ users consuming $z_{\max }$. We pick these among the most deprived users in terms of penalty budget. Note that it might not always be possible to satisfy the quota, since users with depleted penalty budgets must be served at $z_{\max }$. An adaptive quota is left for future study. For now, we simply wish to show that there exist values of the quota $N_{a}^{*}$, such that $\operatorname{QBAP}\left(N_{a}^{*}\right)$ outperforms MCAP.

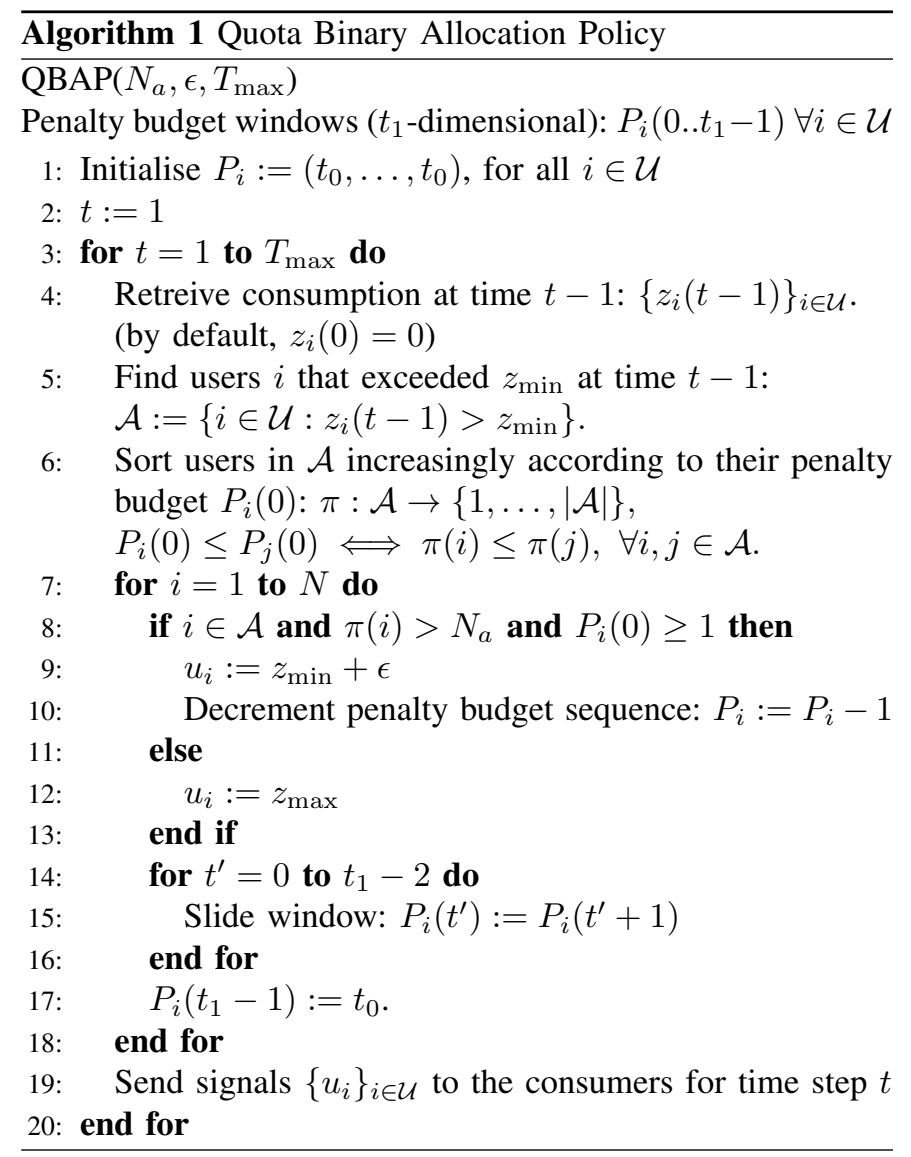




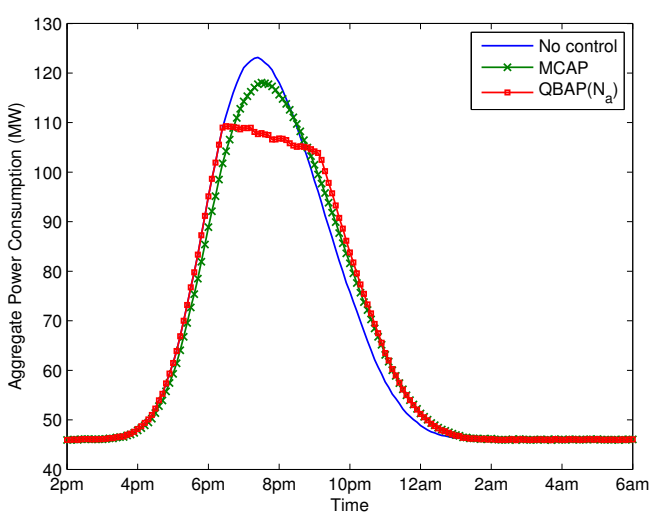

(a) $t_{0}=30 \mathrm{mn}$, quota $N_{a}=6100$

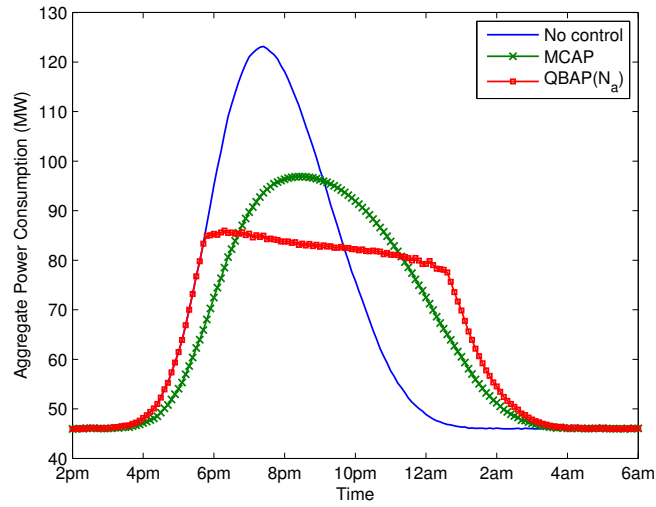

(b) $t_{0}=3 \mathrm{~h}$, quota $N_{a}=3500$.

Fig. 5. The peak-shaving effect of MCAP (Section VI) and QBAP (Section VII). $N=10000$ users, $w=52 \mathrm{kWh}, z_{\max }=14.6 \mathrm{~kW}, z_{\min }=5 \mathrm{~kW}, \epsilon=$ $100 \mathrm{~W}$, time step $=6 \mathrm{mn}$.

\section{Numerical Evaluation}

Customers are guaranteed minimum power of $z_{\min }=5 \mathrm{~kW}$. The non-elastic appliances may consume at most $z_{\min }$ power (e.g., physically limited by a fuse box). Non-elastic consumption level for user $i$ at time $t$ is given by independent normally distributed random variables $X_{i, t}$ of mean $z_{\min }$ and standard deviation 1 capped at $z_{\min }: \min \left\{z_{\min }, X_{i, t}^{+}\right\}$.

The $N$ consumers start charging their batteries at random times normally distributed, centered at $6 \mathrm{pm}$, with standard deviation of 1 hour. We consider identical PEV batteries of capacity $w=52 \mathrm{kWh}$, with charge levels $\left(w_{i}\right)_{i \in \mathcal{U}}$ uniformly distributed between 0 and $4 / 5$ of the total battery capacity $w$. We consider ideal batteries, without losses or charging inefficiencies. The batteries charge at the power imposed by the control signals $z_{\min }+\epsilon$ and $z_{\max }$ (for $\epsilon=100 \mathrm{~W}$ ) without hindering the non-elastic appliances. The maximum power at which the batteries can be charged is $z_{b}=9.6 \mathrm{~kW}$. The overall consumption is capped at $z_{\max }=z_{\min }+z_{b}=14.6 \mathrm{~kW}$.

Under a service curve $\beta_{2}(\cdot)$, battery $i$ may take up to $T_{i}$ time steps to recharge, where $\beta_{2}\left(T_{i}\right)=w_{i}+T_{i} z_{\min }$. A backof-the-envelope calculation gives $T_{i}=\frac{w_{i}}{z_{\max }-z_{\min }}+t_{0}$.

In Figure 5 we show the aggregate consumption for two values of $t_{0}$ : a Voltalis-like setting $t_{0}=30 \mathrm{mn}$ (Figure $5(\mathrm{a})$ ) and a less constraining value $t_{0}=3 \mathrm{~h}$ (Figure 5(b)). We consider two possible controls. In the first case, the PEVs transmit their charge levels $w_{i}$ to the DSO, which in turn implements the smooth $\operatorname{MCAP}\left(T_{i}\right)$ customized for each consumer $i \in \mathcal{U}$. This control gives an aggregated consumption shown by the curves labeled "MCAP". In the second case, the DSO uses QBAP $\left(N_{a}\right)$ for the specified versions of the quota $N_{a}$. This control gives an aggregated consumption shown by the curves labeled "QBAP $\left(N_{a}\right)$ ". For comparison we also plot the aggregate demand when power consumption per user is capped at $z_{\max }$ and no control is used.

We notice that in the considered settings QBAP outperforms MCAP. In the Voltalis-like setting (Figure 5(a)) QBAP reduces peak consumption by $11.3 \%$ (vs. $4.1 \%$ for MCAP). For $t_{0}=$ 3 h (Figure 5(b)), QBAP reduces peak consumption by $30.2 \%$ (vs. $21.3 \%$ for MCAP). Note that the overall energy output (i.e., the integral over time of the consumed power) is the same for the three policies. Moreover, for $t_{0}=3 \mathrm{~h}$ we obtain significant reductions in peak load, while PEV batteries are fully charged by $3 \mathrm{am}$.

\section{CONCLUSION}

We have introduced the concept of service curves for electricity consumption, inspired from load smoothing mechanisms proposed for the Internet. Service curves provide the systems operator with the ability to alleviate the load on the grid at critical peak-hours through demand-response mechanisms. Such mechanisms come at the cost of small discomfort on the consumer side in the form of service reductions over limited periods of time. This approach has the advantage of decoupling the consumer and provider problems, while avoiding to expose consumers to electricity price volatility. We have formally characterized such service curves using max-plus calculus and provided efficient methods to provably implement the constraints imposed thereby. We have shown that on the consumer side, discomfort can be reduced by employing simple policies which take into consideration the parameters of the service curves. Finally, we have provided simple heuristics that can be implemented by the DSO in order to reduce peak load. In the setting of bursty arrivals of heterogeneous consumers, we have shown that QBAP, a heuristic which controls the load via binary power signals, outperforms MCAP, which sends smooth signals and is provably optimal in a stationary setting.

\section{REFERENCES}

[1] S. Borenstein, M. Jaske, and A. Rosenfeld. Dynamic pricing, advanced metering, and demand response in electricity markets. 2002.

[2] S. Keshav and C. Rosenberg. Direct adaptive control of electricity demand. 2010.

[3] J.-Y. Le Boudec and P. Thiran. Network Calculus. Springer Verlag Lecture Notes in Computer Science volume 2050, July 2001.

[4] S. Meyn, M. Negrete-Pincetic, G. Wang, A. Kowli, and E. Shafieepoorfard. The value of volatile resources in electricity markets. In Proc. of the 49th Conf. on Dec. and Control, 2010.

[5] OECD. OECD Economic Surveys: France 2011. OECDiLibrary, March 2011. 